\title{
Turbulent Flow of Water-Based Optimization for WMSNs Based Image Transmission Model
}

\author{
Phani Kulkarni \\ Assistant System Engineer \\ Tata Consultancy Services, Hyderabad, India \\ kulkarniphani@gmail.com
}

\begin{abstract}
The Wireless Sensor Network (WSN) is represented as important in the traditional WSNs and wireless multimedia sensor networks (WMSNs) involvement in the last few decades. The network comprises of small size, minimum power, minimum cost sensor which is referred to as the WSN. Moreover, by exploiting the multihop the communication information is handled and presents merely easy sensing data like temperature, humidity, etc, where WMSNs are indicated as distributed sensing networks that consist of video cameras that comprise sector sense areas. Aforesaid WMSNs can transmit, receive and video information data process is done that is highly demanding and complex by covering with the wireless transceiver. The WMSNs and WSNs deviate regarding their directivity and turnability characteristics. The major contribution of this work is to extend network lifespan with minimized energy utilization by exploiting a developed optimization approach. By optimizing system parameters, optimal transmission radius is attained in order to transfer the sensor information to the resulting SN which is presented within the range. Hence, this work presents a novel optimization technique known as Turbulent Flow of Water-based Optimization (TFWO) for the optimal solution. At last, the developed model performance is examined and evaluated with the conventional models regarding the delay and statistical analysis.
\end{abstract}

Keywords: Alive Nodes, Energy, Optimal Transmission, Video Camera, WSN, WMSNS

\begin{tabular}{ll} 
Nomenclature & \\
\hline Abbreviations & Descriptions \\
\hline WSN & Wireless Sensor Network \\
MIP & Mixed Integer Programming \\
TFWO & Turbulent Flow of Water-based Optimization \\
SN & Sensor Nodes \\
ISH & Indoor Solar Harvester \\
QoS & Quality of Service \\
EC & Error Control \\
EDACR & Efficient Distributed Adaptive Cooperative Routing \\
EETT & Energy Efficient Target Tracking \\
CHS & Cluster Head Selection \\
CS & Compressive Sensing \\
EH & Energy Harvesting \\
RLS & Recursive Least Squares \\
BS & Base Station \\
BCS & Binary Compressive Sensing \\
CEN & Camera Equipped Node \\
\hline
\end{tabular}

\section{Introduction}

In the past few decades, WSN plays an important role in the research community, which shows a wealth in practical and theoretical confronts. In recent times, the accessibility of reasonably priced hardware namely microphones and CMOS cameras which are capable too far and wide capture multimedia content from the environment is promoted WMSNs advancements. In order to retrieve the still images, scalar sensor data, video and audio streams, wireless interlinked network devices are permitted [7]. WMSNs will not merely improve conventional sensor network technologies namely home automation, tracking 
and monitoring of environment however they will additionally enable various novel applications namely potentially appropriate activities storage, multimedia surveillance sensor networks, evasion of Traffic, developed health care delivery, enforcement, and control systems, Automated support for the aged and family monitors, monitor the environmental so on. In both the outdoor as well indoor environments, the wireless camera sensors are exploited, whereas network infrastructure and energy are not present well as whereas no human interference is probable. They present an extensive applications panel whether for industrial, environmental, else military monitoring [1].

In recent days, WMSNs is considered one of the trendy applications in industries because of their several application fields and minimum cost. The WMSNs are used in industrial factories for monitoring applications. For example, using WMSN-based early warning systems, in manufacturing process any failures causes' high repair cost, can be prevented. Besides failure detection, employing WMSNs the consumption of industrial machines' performance and devices can be controlled and monitored to enhance system competence. Moreover, using industrial mobile robots, the environment monitoring applications with multimedia sensors take benefit of WMSNs to protect QoS [2].

To present the real-time data [7] and reliable the WMSN is used for the Internet of-Vehicles, hence the networks QoS, particularly reliability and real-time of it is always focal point while modeling routing protocols for WMSN. For instance, proficient wireless signals transmission, and precise data acquisition, is very important in scenario of imperative circumstances namely traffic accidents, and traffic violations taking into consideration of option that one minute earlier could have reserved victims alive [3].

The major objective of this research is to implement a novel optimization approach; it is used to improve the WMSN lifetime. Here, it is acquired by choosing optimal system parameter, thus optimal transmission radius is acquired for transferring sensor information to corresponding SN, which are within range. Moreover, a novel optimization approach is exploited to select the CH optimally. Finally, the proposed method performance is evaluated with the traditional techniques regarding various metrics.

\section{Literature Review}

In 2020, Denghui Wang et al [1], developed energy- EDACR for WMSN, the Quality of Service and energy utilization constraints were considered. Specifically, a reinforcement learning-based model was designed in order to carry out the energy balanced routing and QoS on the basis of the knowledge of delay and reliability. Finally, the experimentation outcomes exhibit that the utilization of energy was minimized when assuring QoS evaluated with the conventional cooperative protocol.

In 2013, Debashis De et al [2], worked on the WMSN, where, the energy effectual operations were specifically significant to expand the monitoring. Here, the energy-efficient mechanism EETT was developed in that the detection of target ability was maximized using the camera SN rotation in WMSN as it recognizes any target in its view area.

In 2018, Yang Yang et al [3], addressed conflict amid massive data processing and restricted-energy or bandwidth. A 2-layer compressive sensing-based video decoding, and encoding model, was proposed that not merely important minimize the sampling data number. However, from distributed SN to the prevailing base station was transferred. At the initial layer, the initial group sparse was modeled to exploit the correlation amid frames for every sensor node. Next, the spatial correlation among the neighbor nodes was considered. The second group basis was attained using dictionary learning.

In 2020,Nazli Tekin and VehbiCagriGungor [4], worked on the different data size impact reduction techniques, like CS, image compression, and EH techniques, like thermal, vibration, and indoor solar, on the lifespan of WMSNs in industrial environments. Additionally, a new MIP model was presented to increase the lifetime of the network, while the CS, EH, and EC techniques were used together. Finally, the experimental outcomes show that the comparative analysis which utilizes the BCS and ISH expands industrial network lifespan considerably.

In 2019, Sheng Feng et al [5], developed a real-time 3D localization approach comprehending using a distributed model with several smart devices to surmount network unsteadiness and bottleneck channel at coordinator. The RLS method was used to combine 2D images organized from multiple views synchronously in WMSNs in an indoor environment, to ascertain the mobile robot's 3D location. A distributed architecture was developed to reduce the wireless data transmission which integrates several smart devices to define the data content which transmits from several wireless visual sensors. 


\section{Image Transmission In WMSN}

\subsection{Network Model}

In this section, a network model with respect to the camera-equipped and normal SN is demonstrated. Here, the complete nodes do not possess the capability of image capturing and it is not possible particularly in the dense and large WMSNs. In CENs, the first and foremost images are provided and the images are transferred to BS that is distinct from the sensing area. In addition, the number of BS is distantly least in the specific area from general SN due to the higher sensing radii on CENs. Moreover, the radius of transmission is dynamically adjusted using SN which are consists in the network model. In the $\mathrm{SN}$, based on the uneven remaining energy distribution, the modification is done in the transmission of CEN's radius which directs to extend the lifetime of the network.

\subsection{Energy Consumption Model}

Eq. (1) and (2) denotes consumption of energy in transmitting and receiving $p$ bit data on distance $t$.

$$
\begin{aligned}
& \mathrm{EG}_{\mathrm{tm}}(\mathrm{p}, \mathrm{t})= \begin{cases}\mathrm{EG}_{\mathrm{ecl}} \cdot \mathrm{p}+\varepsilon_{\mathrm{fr}} \cdot \mathrm{p} \cdot \mathrm{t}^{2}, & \mathrm{t}<\mathrm{t}_{\mathbf{0}} \\
\mathrm{EG}_{\mathrm{ecl}} \cdot \mathrm{p}+\varepsilon_{\mathrm{mc}} \cdot \mathrm{p} \cdot \mathrm{t}^{4}, & \mathrm{t} \geq \mathrm{t}_{\mathbf{0}}\end{cases} \\
& \mathrm{EG}_{\mathrm{rm}}(\mathrm{p})=\mathrm{EG}_{\mathrm{ecl}} \cdot \mathrm{p}
\end{aligned}
$$

$E G_{e c l}$ refers utilized energy by circuit per bit $t$ denotesdistance amidwireless transmitter and the receiver, $\varepsilon_{f r} t^{2}$ or $\varepsilon_{m c} t^{4}$ represents as amplifier energy. On the basis of the eq. (1) and (2), the utilization of energy among the transmitter and receiver is updated. Furthermore, Eq. (3) indicates the consumption of energy per bit, $E G_{D W E}$ indicates the debauched energy for one level wavelet transformation per bit, $E G_{c o d}$ indicates needed energy for coding and $D L_{0}$ indicates wavelet decomposition level.

$$
\mathrm{EG}_{\mathrm{cpt}}=\mathrm{EG}_{\mathrm{DWE}} \cdot \sum_{\mathrm{DL}=\mathbf{1}}^{\mathrm{DL}_{\mathbf{0}}}\left(\frac{\mathbf{1}}{\mathbf{4}}\right)^{\mathrm{DL}-\mathbf{1}}+\mathrm{EG}_{\mathrm{cod}}
$$

\subsection{Analysis of Energy Consumption Model for CEN}

The utilization of energy to transfer an image size of $P \times Q$ pixels with eight bits per pixel is carried out on the basis of Eq. (4) throughoutCENtransfers raw images to nearby $\mathrm{CH}$.

$t_{C A-C L}$ indicates distance amidCH and a camera-equipped node.

$$
\mathrm{EG}_{\mathrm{CA}}=\mathrm{EG}_{\mathrm{TM}}\left(\mathrm{P} \cdot \mathrm{Q} \cdot \mathbf{8}, \mathrm{t}_{\mathrm{CA}-\mathrm{CL}}\right)
$$

By utilizing the eq. (5), utilization of energy for $X \times Y$ size with 8 bits per pixel is carried out, if the image, which transmits is compressed using a camera-equipped node. Moreover, $B_{R}$ indicates a compressed image bit rate.

$$
\mathrm{EG}_{\mathrm{CA}}^{\prime}=\mathrm{EG}_{\mathrm{cpt}} \cdot \mathrm{P} \cdot \mathrm{Q} \cdot \mathbf{8}+\mathrm{EG}_{\mathrm{TM}}\left(\mathrm{P} \cdot \mathrm{Q} \cdot \mathrm{B}_{\mathrm{R}}, \mathrm{t}_{\mathrm{CA}-\mathrm{CL}}\right)
$$

\subsection{Analysis of Energy Consumption Model for $\mathbf{C H}$}

In several rounds, the operation of clustering is performed. At first, after the Cluster Head Selection (CHS), the set-up phase is carried out. Then the steady phase is performed while the image data transformed is performed on the basis of $C L$ from one node and on pinnacle of BS. Then, in consideration with the camera-equipped node for each node at the steady-state stage, as stated in Eq. (6), if it operates compression against transmitting images, subsequently energy utilization of $\mathrm{CH}$ to increase compressed data and navigating them to BS. $t_{C H-b t}$ signifies distance amid $\mathrm{CH}$ and BS.

$$
\mathrm{EG}_{\mathrm{CL}}=\mathrm{d} \cdot \mathrm{EN}_{\mathrm{RM}}\left(\mathrm{P} \cdot \mathrm{Q} \cdot \mathrm{B}_{\mathrm{R}}\right)+\mathrm{d} \cdot \mathrm{E}_{\mathrm{TM}}\left(\mathrm{P} \cdot \mathrm{Q} \cdot \mathrm{B}_{\mathrm{R}}, \mathrm{l}_{\mathrm{CL}-\mathrm{bt}}\right)
$$

Eq. (7) indicates energy utilization of $\mathrm{CH}$ in obtaining image and it compresses then transfers the compressed image to BS while the CEN transfer image to the Cluster Head and if Cluster Head compress the image.

$$
\begin{gathered}
\mathrm{EG}_{\mathrm{CL}}^{\prime}=\mathrm{d} \cdot \mathrm{EG}_{\mathrm{RM}}(\mathrm{P} \cdot \mathrm{Q} \cdot \mathbf{8})+\mathrm{d} \cdot \mathrm{EG}_{\mathrm{cpt}} \cdot \mathrm{P} \cdot \mathrm{Q} \cdot \mathbf{8}+ \\
\mathrm{d} \cdot \mathrm{EG}_{\mathrm{TM}}\left(\mathrm{P} \cdot \mathrm{Q} \cdot \mathrm{B}_{\mathrm{R}}, \mathrm{t}_{\mathrm{CL}-\mathrm{BT}}\right)
\end{gathered}
$$




\subsection{Set-up phase for Camera Cluster}

In-camera clusters if $\mathrm{CHs}$ are represented as camera-equipped nodes camera cluster set up model functions are estimated only at a single time. In-camera cluster set-up phase, 3 phases are presented they are stated as below:

a) Using a nonpersistent CSMA MAC protocol message is transmitted via a different transmission radius $T X D_{c}$ to statecamera-equipped node presence. In reality, the message is a minute message which engages header and CEN ID, in that message is represented as an information message.

b) The only one that defines that camera cluster needs to concatenate is the SN, which is based on strength of messages received signal from camera nodes adjacent CEN is transferred reverse with a joinrequest messageusing a nonconstant CSMA MAC protocol.In this message, the camera-equipped nodeID is involved, the remaining energy is performed between its distances and the ordinary cluster is GH.

c) Using CEN, transmission radius is adjusted to schedule camera cluster. It is performed in the achievement of the integrated request messages and ascertains image compression task allotment on nodes within groups of the camera, which is based on remaining energy. Subsequent to that, TDMA scheduling is performed and transmits this schedule gone.

\subsection{Determination of $T X D_{c}$}

In the camera cluster setup phase, $T X D_{c}$ determination is considered as one of the important tasks. In this, based on $T X D_{c}$, two cases are presented such as the node scarcity happens in merging the camera cluster while possessing minimum $T X D_{c}$, and subsequently the divergence happens in the other camera cluster while possessing maximum $T X D_{c}$. As a result, the transmissions of image rate may obtain superior to image compression in CEN. Therefore, it suitable $T X D_{c}$ is efficient for CEN to camera cluster set up. Here, the image size which is exploited is $P \times Q \times 8$ and is captured using a camera-equipped node. The reexamination on energy utilization of camera-equipped nodes is offered with the transmission of the raw image is highly efficient, rather than working on image compression, when $E G_{c}<E G_{c}^{\prime}$. Eq. (8) comprises the definite constraints whicharesatisfiedexploiting $T X D_{c}$.

$$
\mathrm{TXD}_{\mathrm{c}}<\mathrm{TXD}^{\prime}= \begin{cases}\sqrt{\frac{\mathrm{rEG}_{\mathrm{cpt}} /(\mathrm{txd}-\mathbf{1})-\mathrm{EG}_{\mathrm{ecl}}}{\varepsilon_{\mathrm{fr}}},} & \mathrm{TXD}^{\prime}<\mathrm{t}_{\mathbf{0}} \\ \sqrt[4]{\frac{\mathrm{rEN}_{\mathrm{cpt}} /(\mathrm{txd}-\mathbf{1})-\mathrm{EN}_{\mathrm{ecl}}}{\varepsilon_{\mathrm{mc}}}}, & \mathrm{TXD}^{\prime} \geq \mathrm{t}_{\mathbf{0}}\end{cases}
$$

Whereas the ratio of image compression $\left(r=8 / B_{R}\right.$ and $\left.r>1\right)$ is indicated as $r$. Thus, by exploiting the eq. (9), assessment of fixed $T X D_{c}$ is performed, whereas the system parameter which is linked to normal SN density is indicated as $\alpha$, the value of $\alpha<1$.

$$
\mathrm{TXD}_{\mathrm{c}}=\alpha \mathrm{TXD}^{\prime}
$$

Presume that $\rho$ as node density in the monitoring area. In-camera cluster when there are $h_{0}$ nodes, subsequently $\rho \pi T X D_{c}^{2}=h_{0}$. The calculation of system parameter $\alpha$ calculation is stated in eq. (10).

$$
\alpha=\sqrt{\frac{\mathrm{h}_{\mathbf{0}}}{\pi \rho}} / \mathrm{TXD}^{\prime}
$$

One of the important contributions of it is on share image compression task using nodes that happened in camera cluster. The lifespan of the network is maximized by eradicating the common nodes and CEN emerged in the cluster during a similar instant. Let, eq. (11) states the remaining energy of entire nodes is equivalent, which is stated as $R E$, whereas $t_{i}$ indicates distance among the normal nodes to $\mathrm{CH}$ in the common cluster.

$$
\begin{aligned}
& \frac{\mathrm{RE}}{\left\{\mathrm{EG}_{\mathrm{ecl}}+\left(\varepsilon_{\mathrm{fr}} \cdot \mathrm{TXD}_{\mathrm{c}}^{\mathbf{2}}, \varepsilon_{\mathrm{mc}} \cdot \mathrm{TXD}_{\mathrm{c}}^{\mathbf{4}}\right)\right\} \cdot \mathrm{P} \cdot \mathrm{Q} \cdot \mathbf{8}} \\
& =\frac{\mathrm{h}_{\mathbf{0}} \mathrm{RE}}{\mathrm{EG}_{\mathrm{ecl}} \cdot \mathrm{P} \cdot \mathrm{Q} \cdot \mathbf{8}+\mathrm{EG}_{\mathrm{cpt}} \cdot \mathrm{P} \cdot \mathrm{Q} \cdot \mathbf{8}+\left\{\mathrm{EG}_{\mathrm{ecl}}+\left(\varepsilon_{\mathrm{fr}} \cdot \mathrm{t}_{\mathrm{i}}^{\mathbf{2}}, \varepsilon_{\mathrm{mc}} \cdot \mathrm{t}_{\mathrm{i}}^{4}\right)\right\} \cdot \mathrm{P} \cdot \mathrm{Q} \cdot \mathbf{8} / \mathrm{r}}
\end{aligned}
$$

Hence, Eq. (12) indicates estimated optimal node numbers in the camera cluster. 


$$
\mathrm{h}_{\mathbf{0}} \approx\left[\frac{\mathrm{EG}_{\mathrm{cpt}}+\mathrm{EG}_{\mathrm{ecl}}}{\mathrm{EN} \mathrm{ecl}_{\mathrm{el}}}\right]+\mathbf{1}
$$

$\alpha$ indicates system parameter, which is attained by substituting in Eq. (10) to the Eq. (12). Hence, using eq. (9), the fixed transmission radius $T X D_{c}$ is devised.

\subsection{Transmission Radius of Adaptation}

As the wide spread SN remaining energy which concatenates camera cluster is imbalanced, $h_{0}$ exploited in Eq. (12) which is not included as the real optimum value. By broadcasting with $T X D_{c}$ the join-request messages back $h_{0}$ cannot make sure. Hence, using a camera-equipped node the best value $\alpha$ is required to ascertain. Let $R E_{i},\{i=1,2, \ldots h\}$ is represented as the remaining energy of wide spread SN. The estimation of the expected lifespan of $c_{i}$ node is explained in eq. (13).

$$
\beta_{\mathrm{i}}=\left[\frac{\mathrm{RE}_{\mathrm{i}}}{\mathrm{EG}_{\mathrm{rm}}(\mathrm{P} \cdot \mathrm{Q} \cdot \mathbf{8})+\mathrm{EG}_{\mathrm{cpt}} \cdot \mathrm{P} \cdot \mathrm{Q} \cdot \mathbf{8}+\mathrm{EG}_{\mathrm{tm}}\left(\mathrm{P} \cdot \mathrm{Q} \cdot \mathbf{8} / \mathrm{r}, \mathrm{t}_{\mathrm{i}}\right)}\right]
$$

Whereas, the distance between the $\mathrm{CH}$ and node $c_{i}$ in the normal cluster is stated as $t_{i}, r$ indicates the ratio of image compression. Here, to receive an image the node $c_{i}$ 's total dissipated energy from the node (camera-equipped) is stated as denominator value. The image compression and compact data to $\mathrm{CH}$ transmission are stated as a task in the normal cluster.

Consider, $R E_{\text {cam }}$ indicates the CEN $b$ 's remaining energy. Eq. (14) exhibits an expected lifespan of $b$.

$$
\beta_{\mathrm{cam}}=\left[\frac{\mathrm{RE}_{\mathrm{cam}}}{\mathrm{EG}_{\mathrm{tm}}\left(\mathrm{P} \cdot \mathrm{Q} \cdot \mathbf{8}, \mathrm{TXD}_{\mathrm{c}}\right)}\right]
$$

Toincreasenetwork lifespan, common SN, and node (camera-equipped),happens in the cluster needs toexpiresimultaneously, hence, the eq. (15) is attained.

$$
\sum_{\mathrm{t}\left(\mathrm{c}_{\mathrm{i}}, \mathrm{b}\right) \leq \mathrm{RXD}_{\mathrm{c}}}=\beta_{\mathrm{cam}}
$$

\section{Network Life Enhancement}

\subsection{Objective Model}

Conversely, Eq. (15) will not be fulfilled in most cases. Therefore, the transmission radius adjustment in clusters needs to be performed using CEN at any cost which is stated in Eq. (16).

$$
\left\{\begin{array}{cc}
\min & \sum \beta_{\mathrm{i}} \\
\mathrm{t}\left(\mathrm{c}_{\mathrm{i}}, \mathrm{b}\right) & \leq \mathrm{TXD}_{\mathrm{c}} \\
& \sum \beta_{\mathrm{i}} \geq \beta_{\mathrm{cam}} \\
\mathrm{t}\left(\mathrm{c}_{\mathrm{i}}, \mathrm{b}\right) \leq \mathrm{TXD}_{\mathrm{c}}
\end{array}\right.
$$

Eq. (16) states the developed technique objective model, whereas the network lifetime maximizes. One more objective function is the detection of the optimal $\mathrm{CH}$, is $\mathrm{CH}$. By exploiting the developed model, aforesaid objectives are fulfilled.

\subsection{Proposed Optimization TFWO Model}

Whirlpool is an arbitrary nature behavior that happens in seas, rivers, and oceans. In this paper, various whirlpools are used (or various groups of whirlpools with the center of an optimal member of the group as the hole of the whirlpool, that posses the role of using centripetal force on the other members), that capture the particles and objects around them based on their distances and suck them and attempt to preserve them right in the center [6].

In a circular path, that objects move for an object with constant velocity, the circle radius is equivalent to the multiplication of the mass of the object and squared velocity divided by the centripetal force. Moreover, the centrifugal force, and mass also communication among nearby whirlpools and objects occurs resistance and deviation in this suction and traction. 


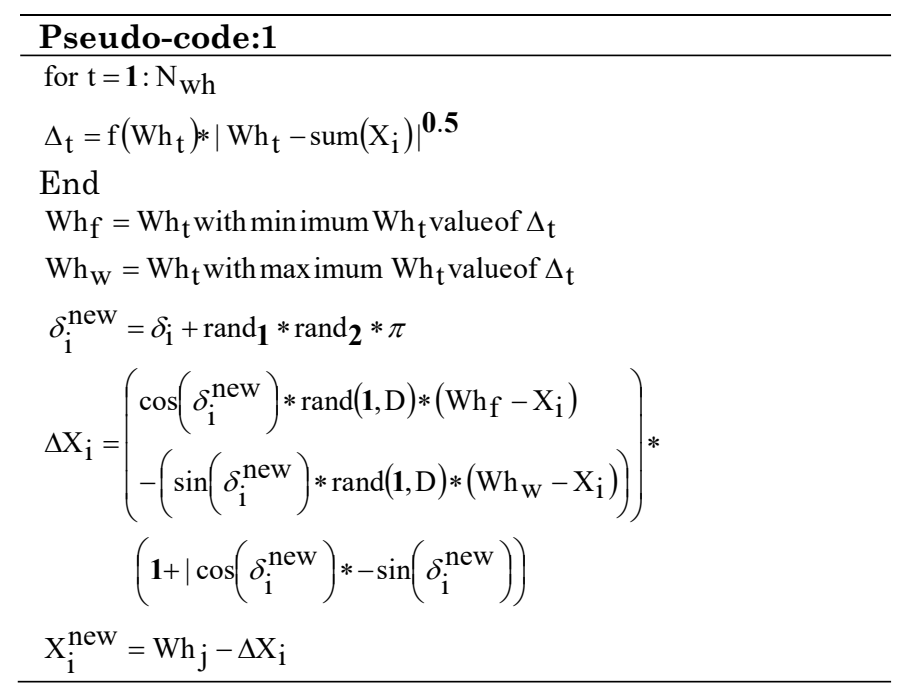

Pseudocode 1 and 2 represents the whirlpools effects on objects of its setting and other whirlpools. Here, $N_{w h}$ represents the groups or whirlpool sets, $W h$ represents whirlpool, $X$ represents objects.

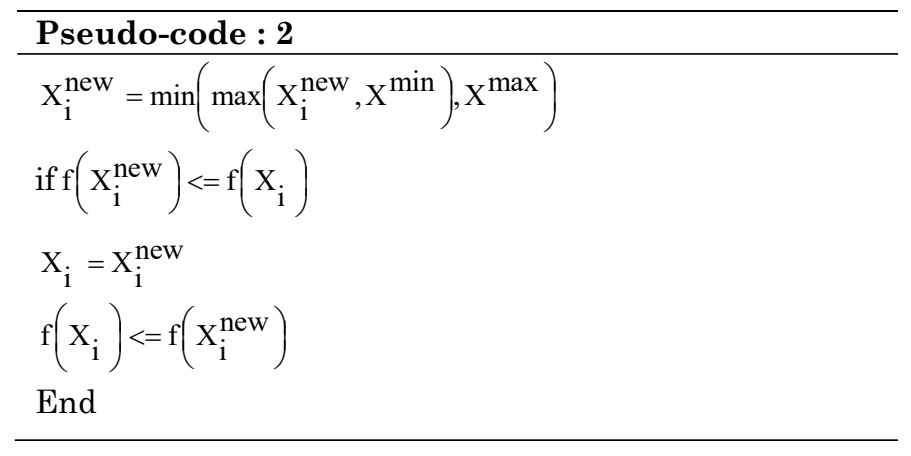

\begin{tabular}{l}
\hline Pseudo-code : $\mathbf{3}$ \\
$\mathrm{FE}_{\mathrm{i}}=\left(\cos \left(\delta_{\mathrm{i}}^{\text {new }}\right)\right)^{2} *\left(\left(\sin \left(\delta_{\mathrm{i}}^{\text {new }}\right)\right)^{2}\right)$ \\
$\mathrm{IF}$ rand $<\mathrm{FE}_{\mathrm{i}}$ \\
$\mathrm{p}=$ round $(\mathbf{1}+$ rand $*(\mathrm{D}-\mathbf{1}))$ \\
$\mathrm{f}\left(\mathrm{X}_{\mathrm{i}}\right)<=\mathrm{f}\left(\mathrm{X}_{\mathrm{i}}^{\text {new }}\right)$ \\
End
\end{tabular}

Pseudocode 3 represents the centrifugal force, where $F E_{i}$ represents the centrifugal force, $\delta$ represents the hole angle Pseudo code 4 and 5 represents the communication among the whirlpools 

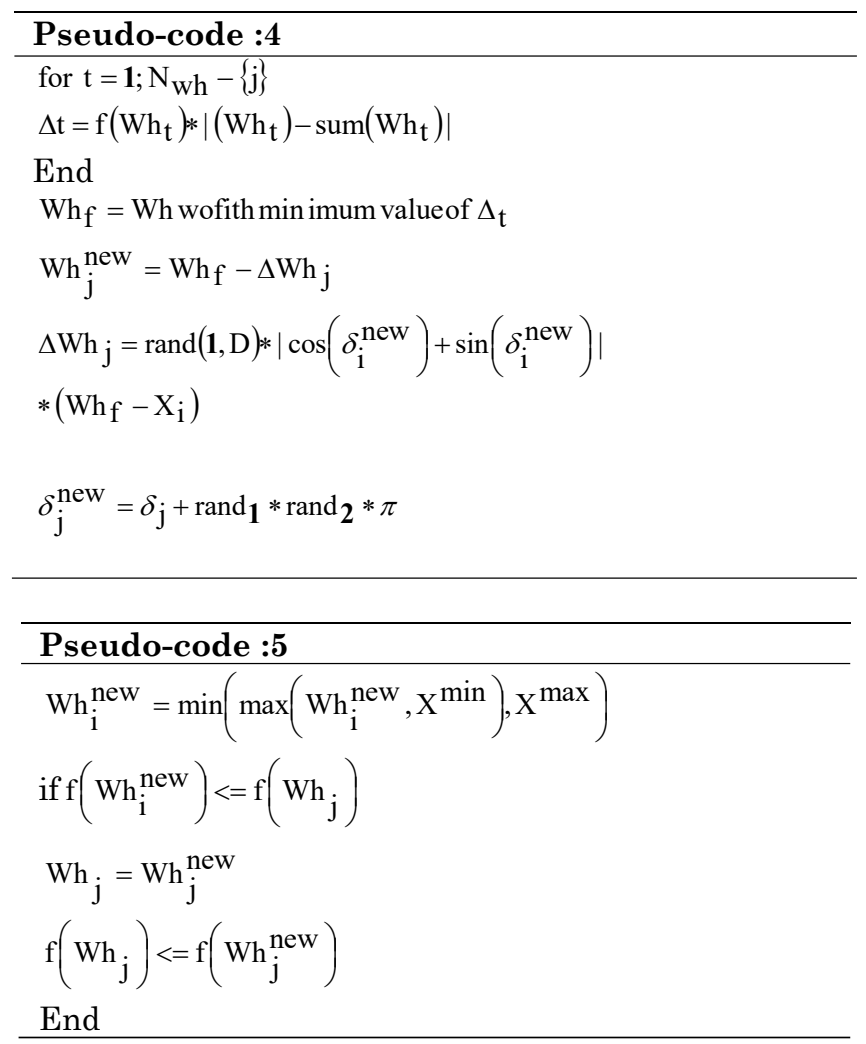

At last, if strongest member between new members of the whirlpool's set has high strength than its equivalent whirlpool, subsequently, it is selected as a new whirlpool for the subsequent iteration.

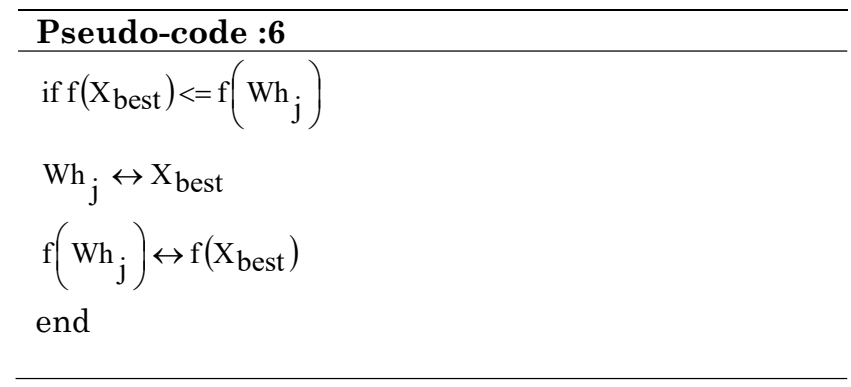

\section{Result and Discussion}

In this paper, the developed model and the conventional models were simulated in this experiment, there were 100 nodes presented in the network and one of the nodes was CEN. In the experimentation model, the network area was $100 \times 100$. The proposed method performance was evaluated with the conventional models regarding several parameters such as delay and statistical analysis. Moreover, the proposed method is compared with the conventional models such as Artificial Bee Colony (ABC), Particle Swarm Optimization (PSO), Grey Wolf Optimization (GWO), Whale Optimization Algorithm (WOA), and Genetic Algorithm (GA).

Fig 1 demonstrated the analysis of the adopted and traditional techniques. Here, the rounds are presented in the range 0 to 2000. The developed technique and the performance of the existing technique over the delay are exhibited in Fig 2. From Fig 2, it is very evident that the developed technique has a minimum delay than any other existing techniques in turns maximizes the network lifetime.

Fig 3 demonstrates the statistical analysis of developed and existing techniques regarding their network energy level. From the analysis from the figure, it is evident that the developed technique 
attained higher network energy while comparing with the traditional techniques. The explanation for each and every case is standard deviation, mean best case, median and worst case. Therefore, the developed technique exhibits superior performance regarding maximum energy.

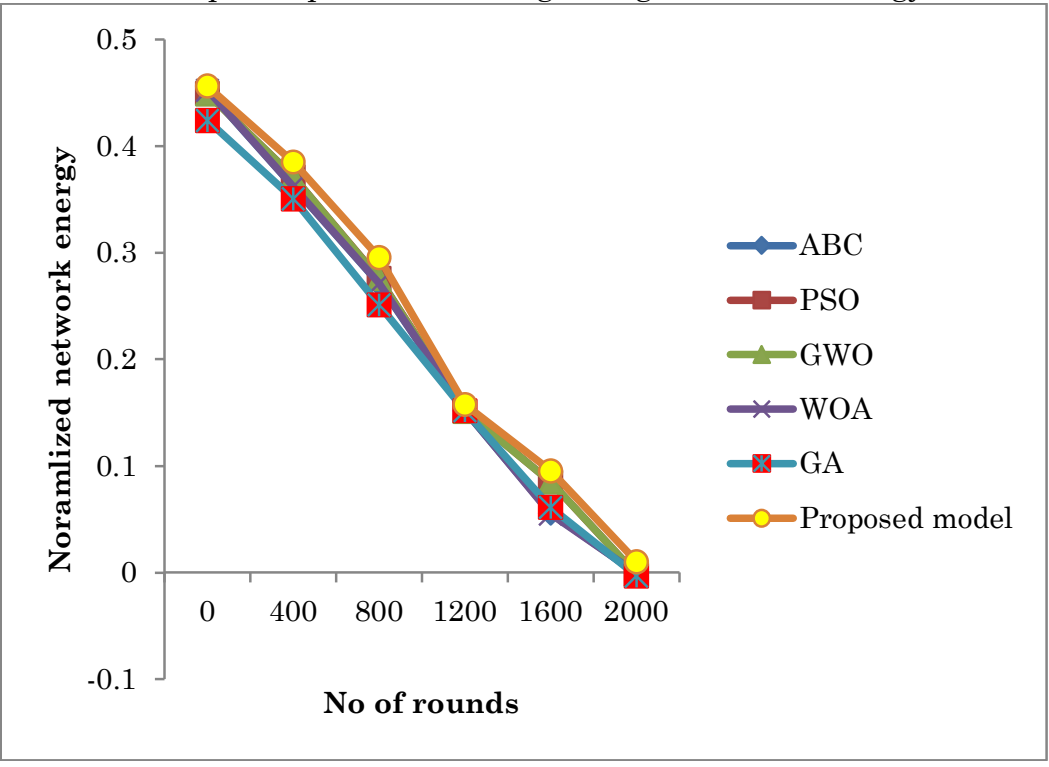

Fig. 1. Analysis of adopted and traditional models concerning normalized network energy

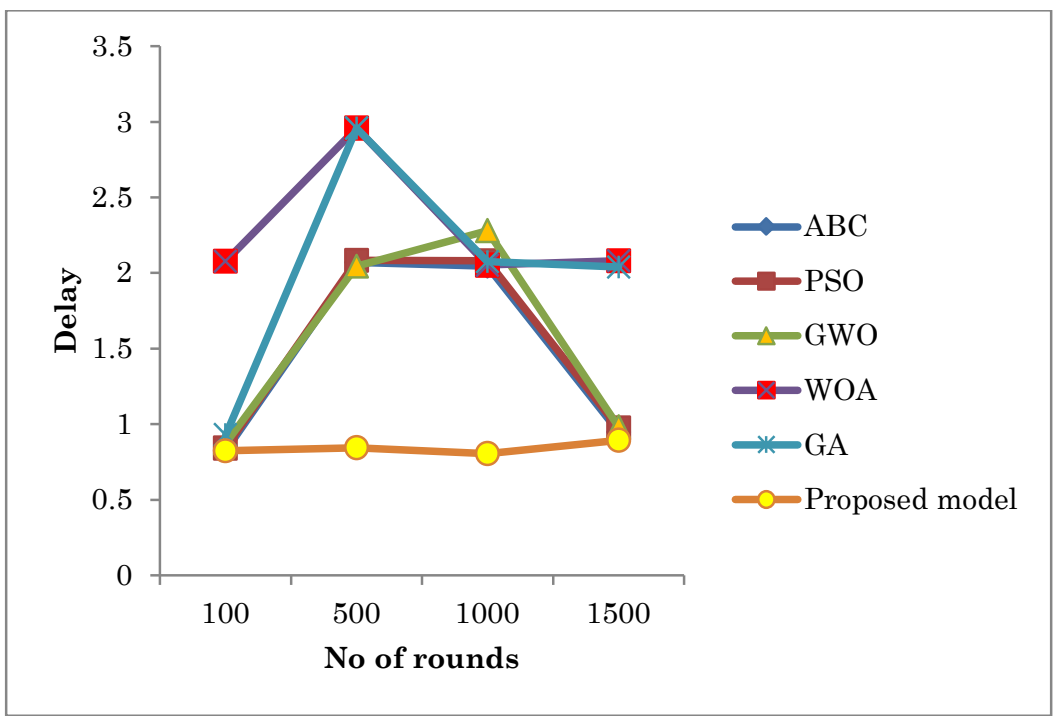

Fig. 2. Analysis of adopted and traditional models concerning delay 


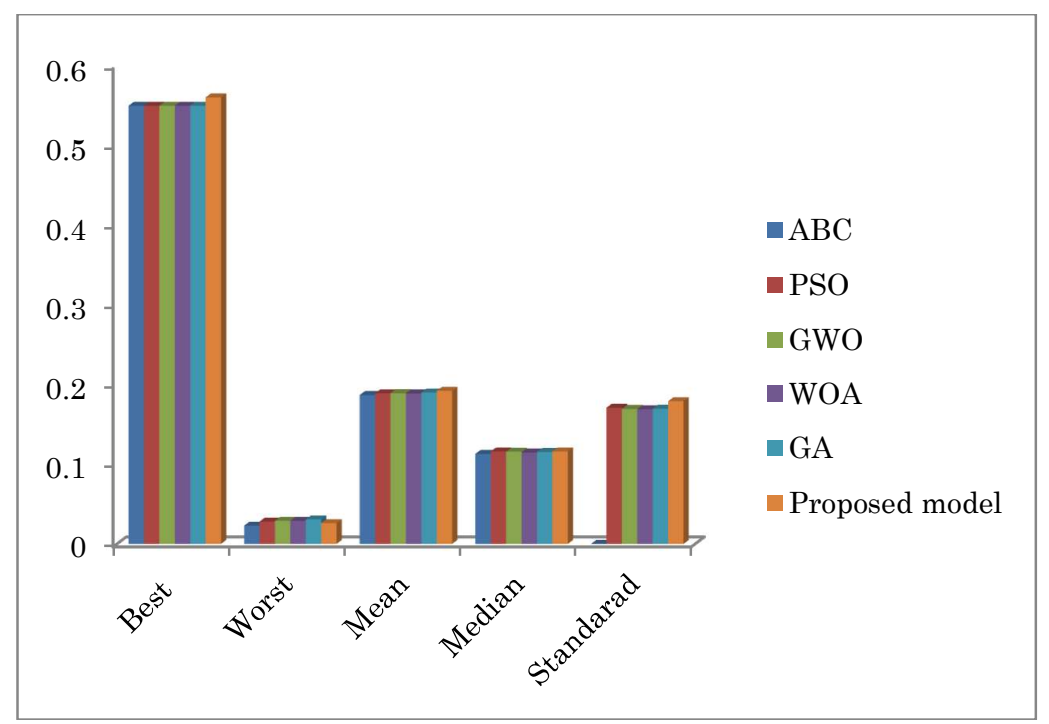

Fig. 3. Statistical Analysis of adopted and traditional models

\section{Conclusion}

This paper presented a novel approach $\mathrm{CH}$ with CEN that helps for the extension of the network lifetime by exploiting the optimization algorithm. The system parameter optimization was performed thus the optimal transmission radius was attained by exploiting the sensor information broadcast to the equivalent SN that are collected within the range. Furthermore, a novel approach named TFWO was introduced in this work. Subsequent to that, the $\mathrm{CH}$ optimal selection was carried out by exploiting the developed method. At last, the developed technique performance was evaluated with the traditional methods regarding the delay and statistical analysis.

\section{Compliance with Ethical Standards}

Conflicts of interest: Authors declared that they have no conflict of interest.

Human participants: The conducted research follows the ethical standards and the authors ensured that they have not conducted any studies with human participants or animals.

\section{References}

[1] Denghui WangJian LiuDezhong Yao,"An energy-efficient distributed adaptive cooperative routing based on reinforcement learning in wireless multimedia sensor networks", Computer Networks, vol. 178, 24 May 2020.

[2] Debashis DeMadhuparna Das GuptaAditi Sen,"Energy Efficient Target Tracking Mechanism using Rotational Camera Sensor in WMSN", Procedia Technology, vol. 6, pp. 674-681, 2012.

[3] Yang YangSongtao GuoYuanyuan Yang,"Two-layer compressive sensing based video encoding and decoding framework for WMSN", Journal of Network and Computer Applications, vol. 117, pp. 72-85, May 2018.

[4] Yang YangSongtao GuoYuanyuan Yang,"Two-layer compressive sensing based video encoding and decoding framework for WMSN", Journal of Network and Computer Applications, vol. 117, pp. 72-85, 29 May 2018.

[5] Sheng FengShigen ShenYunzhou Zhang,"Three-dimensional robot localization using cameras in wireless multimedia sensor networks", Journal of Network and Computer Applications27 August 2019.

[6] Mojtaba GhasemiIraj Faraji DavoudkhaniLi Li,"A novel and effective optimization algorithm for global optimization and its engineering applications: Turbulent Flow of Water-based Optimization (TFWO)", Engineering Applications of Artificial Intelligence, vol. 92, 6 May 2020.

[7] K.Srinivas,"Prediction of E-Learning Efficiency by Deep Learning in EKhool Online Portal Networks", Multimedia Research, vol. 3, no. 4, October 2020.

[8] Arvind Madhukar Jagtap,"Developing Deep Neural Network for Learner Performance Prediction in EKhool Online Learning Platform",Multimedia Research, vol. 3, no. 4, October 2020. 